\title{
Proyecto 100/100: experiencia de modelado paramétrico como una opción adaptable de BIM para paises en desarrollo.
}

$100 / 100$ Project: an experience of parametric modeling as a flexible option of BIM for developing countries.

\author{
> Diego Alejandro Velandia R \\ Universidad de los Andes, Colombia. \\ dvelandi@uniandes.edu.co
}

\begin{abstract}
Quality observatory of new housing was created by a collaborative effort between the University of the Andes and Prodesa (building company). From 2005 to 2014 about 800 new housing projects have been evaluated whose results evidenced quality problems. In 2013, a new housing project, which integrated quality criteria from the quality observatory, was commissioned to Universidad de los Andes by Prodesa. Parametric modeling was proposed and applied as a useful tool in the variables integration and the decision making process, to integrate and guarantee quality.
\end{abstract}

\section{Introducción}

En el año 2005 se firma un convenio de cooperación, entre el grupo de investigación GIV de la Facultad de Arquitectura y Diseño de la Universidad de Los Andes y Prodesa (empresa constructora), para iniciar el 'observatorio de calidad para vivienda nueva en Bogotá y la sabana'. Entre los objetivos de este convenio estaban: 1) recopilar y analizar información de proyectos de vivienda nueva en Bogotá, 2) establecer componentes de análisis e indicadores de calidad de la vivienda, tanto en aspectos urbanos como individuales y 3) elaborar el diseño de un sistema que permita administrar la información recopilada como posible herramienta para la toma de decisiones por parte de promotores como de usuarios en proyectos de vivienda nueva.

Entre el ańo 2005 y 2014 se han analizado aproximadamente 800 proyectos de vivienda nueva, a partir de un sistema de indicadores de calidad, que integra aspectos relacionados con las características urbanas, características de la agrupación, características de la unidad de vivienda, aspectos técnicos y acabados. Si bien, el puntaje máximo a obtener en el análisis es 100, el promedio de puntaje para vivienda nueva en el año 2009 era de 44.7, lo que evidenciaba problemas de calidad.

En el año 2013 y con base en los indicadores de calidad existentes, y los resultados obtenidos de los análisis hechos en el observatorio, Prodesa le plantea al Departamento de Arquitectura invertir el proceso. Es decir, no aplicar los indicadores a un proyecto ya construido, y por el contrario promover un proyecto que desde su etapa de diseño integre los indicadores de calidad.
El nombre para este proyecto fue 'Proyecto $100 / 100$ ' por la intención de llegar al puntaje máximo en el proceso de evaluación de vivienda nueva, con un presupuesto máximo de 100 salarios mínimos legales (aproximadamente us $\$ 30.000$ para el año 2013). Por común acuerdo entre las partes, al Departamento de Arquitectura de la Universidad de Los Andes, se le encarga desarrollar el anteproyecto, para que luego, éste sea desarrollado en detalle y construido por Prodesa.

Desde el punto de vista digital, el proyecto planteaba en su momento como problema o reto principal, que la herramienta o metodología de trabajo a utilizar, debería permitir la integración de un número considerable de variables, transmitir la información de forma gráfica e integrar un número importante de profesionales asesores del proyecto. Esta herramienta o metodología debería también permitir que el grupo de profesionales vinculados, pudieran analizar el proyecto y tomar decisiones en una etapa de pre-factibilidad.

La hipótesis que se plantea es que el modelado paramétrico puede ser una herramienta eficaz, capas de proveer una solución a cada uno de los planteamientos anteriores (Figura 1).

\section{Indicadores de Calidad y Concepto de Vivienda}

Para evaluar la calidad en vivienda, primero debemos definir qué tipo de vivienda es la que estamos esperando que se desarrolle y en qué contexto. La vivienda que se está abordando desde el observatorio, es vivienda implantada en un contexto urbano. Una vivienda que va más allá del ámbito estrictamente privado (Montaner, 2010), a partir de cuatro paredes y un techo (ONU, 2010), 


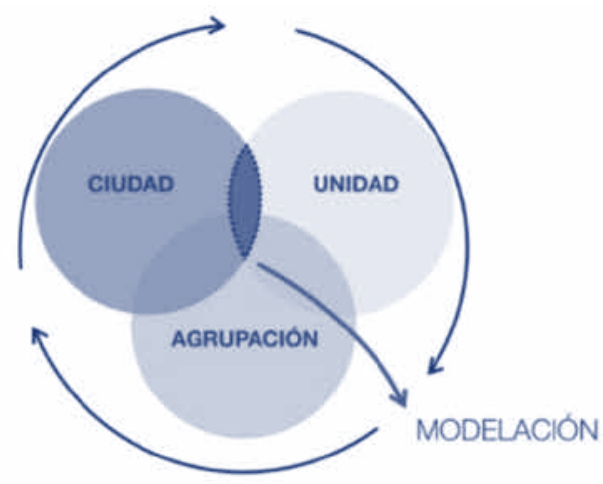

\section{MODELO SISTÉMICO}
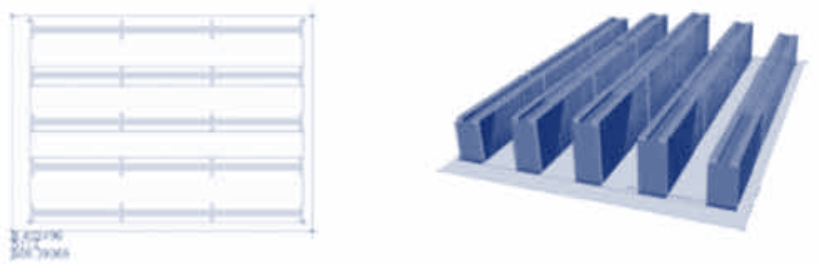

Figura 1: Estrategia de trabajo implementada en proyecto 100/100. Imagen tomada del Documento Técnico de soporte Proyecto 100/100

Por tanto, es una vivienda que además de cumplir con unas condiciones mínimas de habitabilidad, está rodeada de unas condiciones urbanas a diferente escala, que permiten el adecuado desarrollo de la vida cotidiana (Montaner, 2010), en términos de proximidad a equipamientos, servicios y comercio, de sostenibilidad económica, social, ambiental y cultural, y de existencia de elementos y espacios que propicien el encuentro, la convivencia, socialización y demás actividades necesarias para una adecuada vida en comunidad.

A partir de este concepto de vivienda, se definen indicadores cuantitativos que permitan hacer una evaluación lo más objetiva posible. Por eficiencia en el proceso de evaluación, estos indicadores se agruparon en tres escalas espaciales o ámbitos: Ciudad, agrupación y unidad. Cada escala tiene un número determinado de indicadores que buscan verificar si se cumple, y en qué medida, los diferentes aspectos mencionados en la definición del concepto de vivienda (figura 1).

\section{Metodología}

Una de las características de las herramientas digitales aplicadas al campo de la arquitectura, y específicamente del modelado paramétrico, es la complejidad. Es decir, son capaces de integrar un gran número de variables, establecer relaciones complejas entre ellas y permitir realizar análisis compuestos a partir de la modificación de dichas variables (Velandia, 2009). Todo esto además, es posible visualizar en una interfaz gráfica, lo que permite generar discusiones con profesionales que no necesariamente están familiarizados con temas digitales.

Estos argumentos llevaron a definir el uso de Rhinoceros y el plug-in Grasshopper, como herramienta para modelar paramétricamente el proyecto.

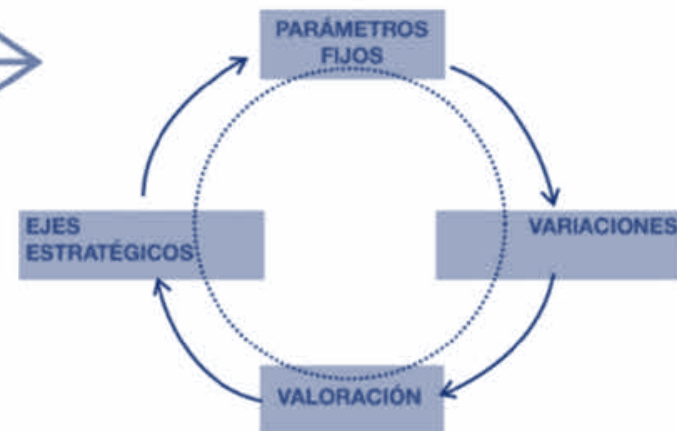

ESTRATÉGIA CÍCLICA
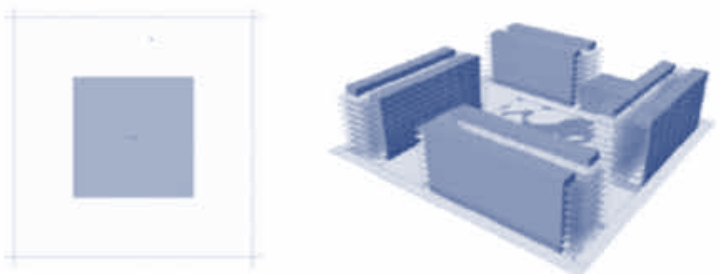

En resumen, se puede hablar de un proceso de trabajo que no fue lineal. Por el contrario, se generaron bucles a partir de someter a discusión los primeros modelos paramétricos, cuyos resultados retroalimentaban el proceso para ir evolucionando el modelo digital (Figura 2).

Se pueden distinguir los siguientes momentos:

1. Definición por parte de Prodesa de un sector para el desarrollo del proyecto y de un grupo interdisciplinar de trabajo: (arquitecto urbanista, experto en presupuesto, proyectistas, etc)

2. Definición por parte del Departamento de Arquitectura de un grupo encargado del proyecto y de un grupo interdisciplinar de asesores (directores, arq. supervisora, profesional de apoyo, monitores, arq. responsable del modelado paramétrico, y eventuales expertos en vivienda, desarrollo estructural, ambiental, gestión, diseño arquitectónico y mobiliario).

3. Análisis de los indicadores de calidad desarrollados por el Observatorio de Vivienda: Se busca convertir los diferentes indicadores en parámetros medibles. A cada parámetro se le asigna un rango donde deben ubicarse para garantizar calidad (Ej. El parámetro de ocupación de estableció máximo hasta el $40 \%$ del área neta urbanizable). Gran número de los indicadores se pudieron parametrizar, sin embargo quedó un grupo que aunque no se parametrizó, se incluyó en el proceso de diseño (Ej. Actividad en primer piso fue un indicador importante en el proceso de diseño, pero no fue susceptible de parametrizar en este caso). Este proceso fue importante para empezar el proceso de diseño como tal, y simultáneamente el proceso de modelado paramétrico. En este punto es donde se empiezan a construir los primeros escenarios, para llevarlos a las discusiones entre los diferentes actores del proceso (Figura 2). 

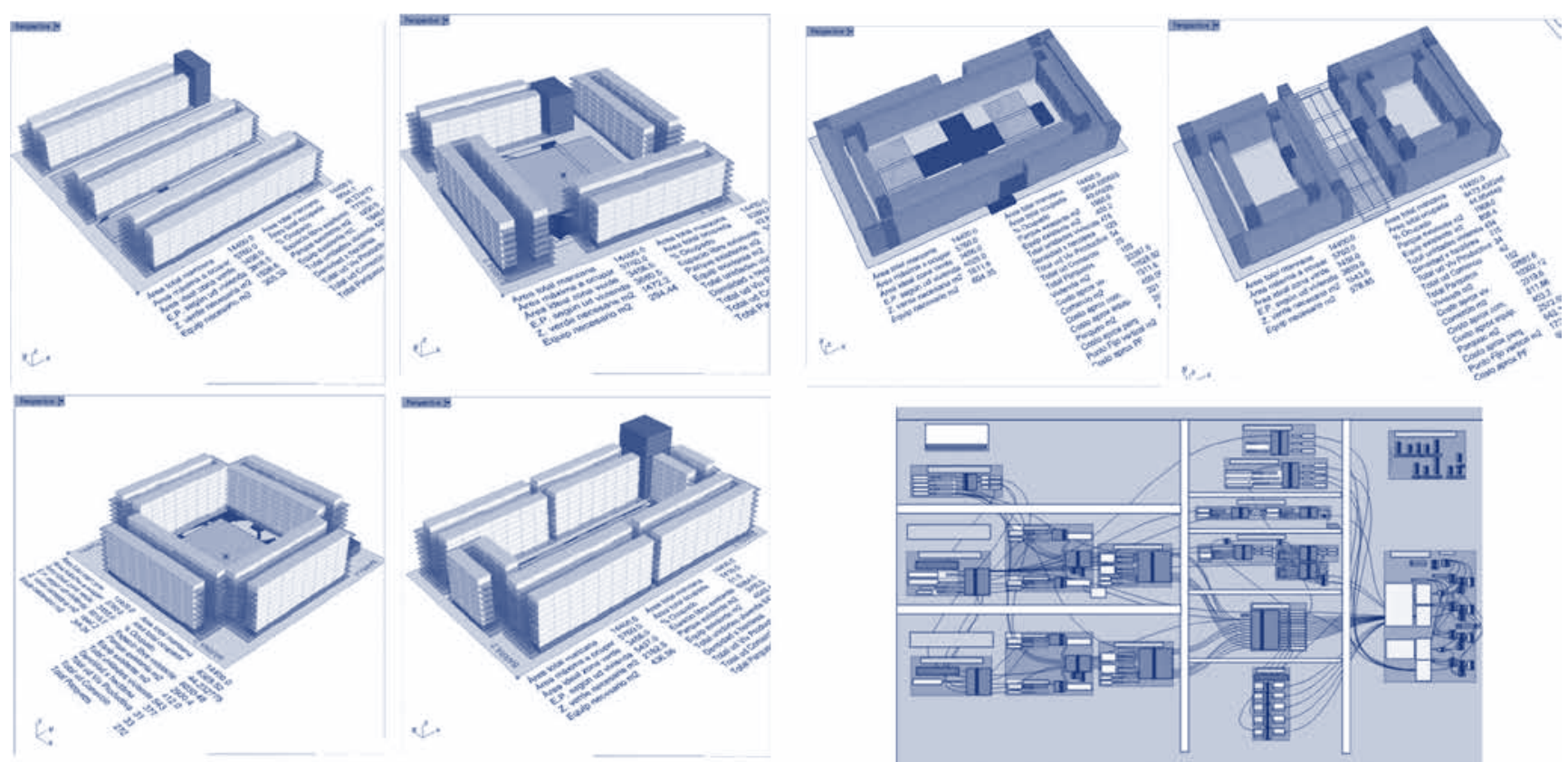

Figura 2: Evolución de los escenarios paramétricos. Visualización gráfica y de datos. Código fuente en grasshopper.

4. Implantación y ocupación: Estos dos temas no se parametrizaron por su complejidad. Se trabajaron a partir de las discusiones con los grupos de trabajo y los asesores, cuyas conclusiones se iban integrando al modelo paramétrico, a partir de la generación de diferentes escenarios, con los cuales se retroalimentaba la discusión y se tomaban nuevas decisiones. En todos los escenarios se revisaba paralelamente, el cumplimiento de los rangos establecidos para los parámetros definidos.

5. Definición de parámetros de análisis (parámetros de salida) útiles al momento de realizar discusiones con el

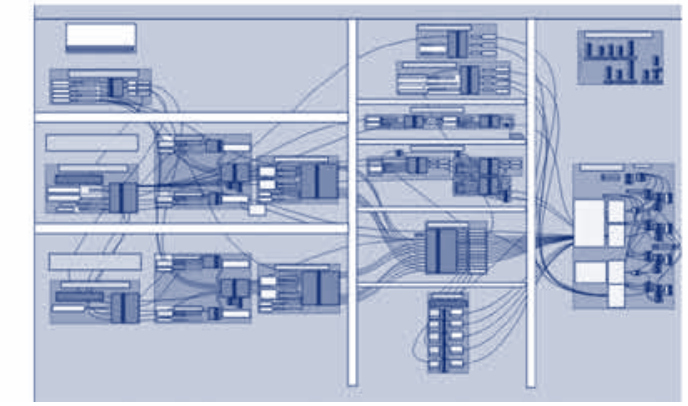

grupo asesor (toma de decisiones). Se pueden distinguir tres tipos de parámetros que se visualizan en los diferentes escenarios: 1) Parámetros que se visualizan gráficamente, los cuales permiten hacerse una idea general de implantación volumetría, escala, relaciones, etc. 2) Parámetros numéricos con el rango ideal a cumplir. 3) Parámetros numéricos con el valor real en cada escenario. Se comparaba el rango ideal y el real para evaluar el nivel de cumplimiento (Figura 3).

Figura 3: Aplicación del código fuente a diferentes escenarios y diferentes geometrías. Más que un proyecto específico es una metodología replicable en diferentes contextos. Imagen tomada del Documento Técnico de soporte Proyecto 100/100.

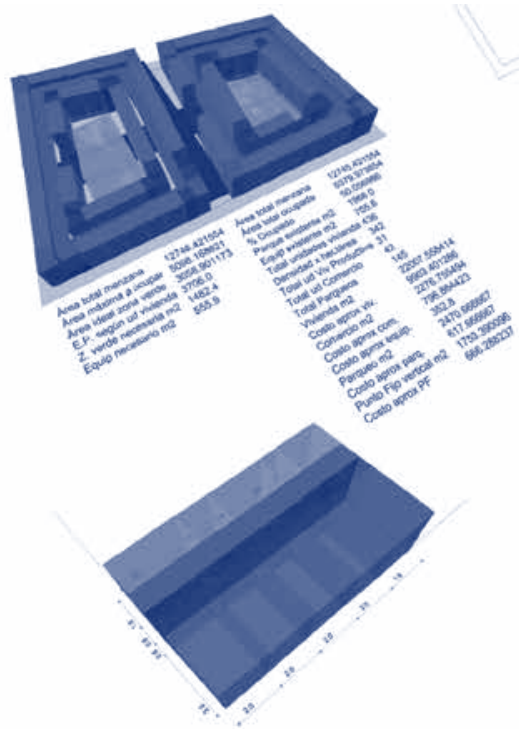

Escenario final paramétrico escala agrupación y unidad
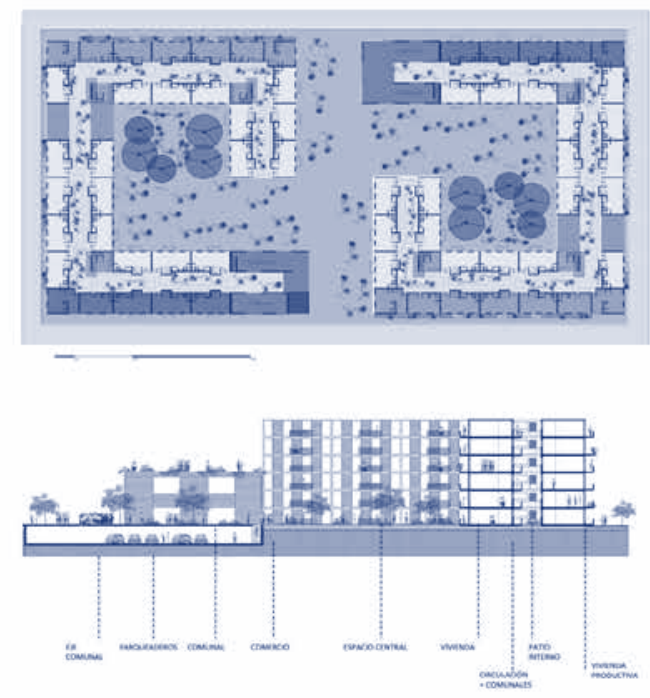

Desarrollo en detalle escala agrupación
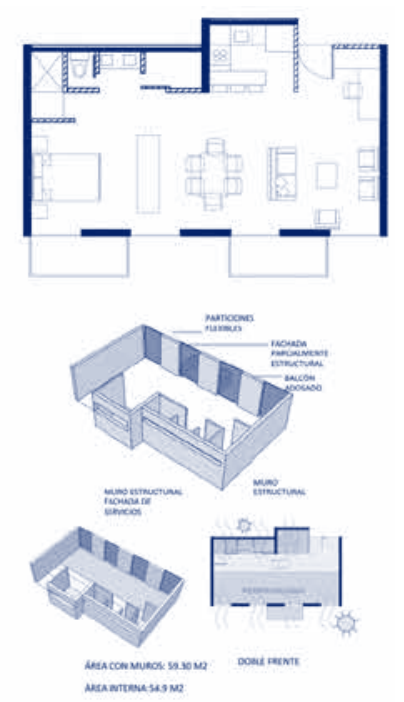

Desarrollo en detalle escala unidad 

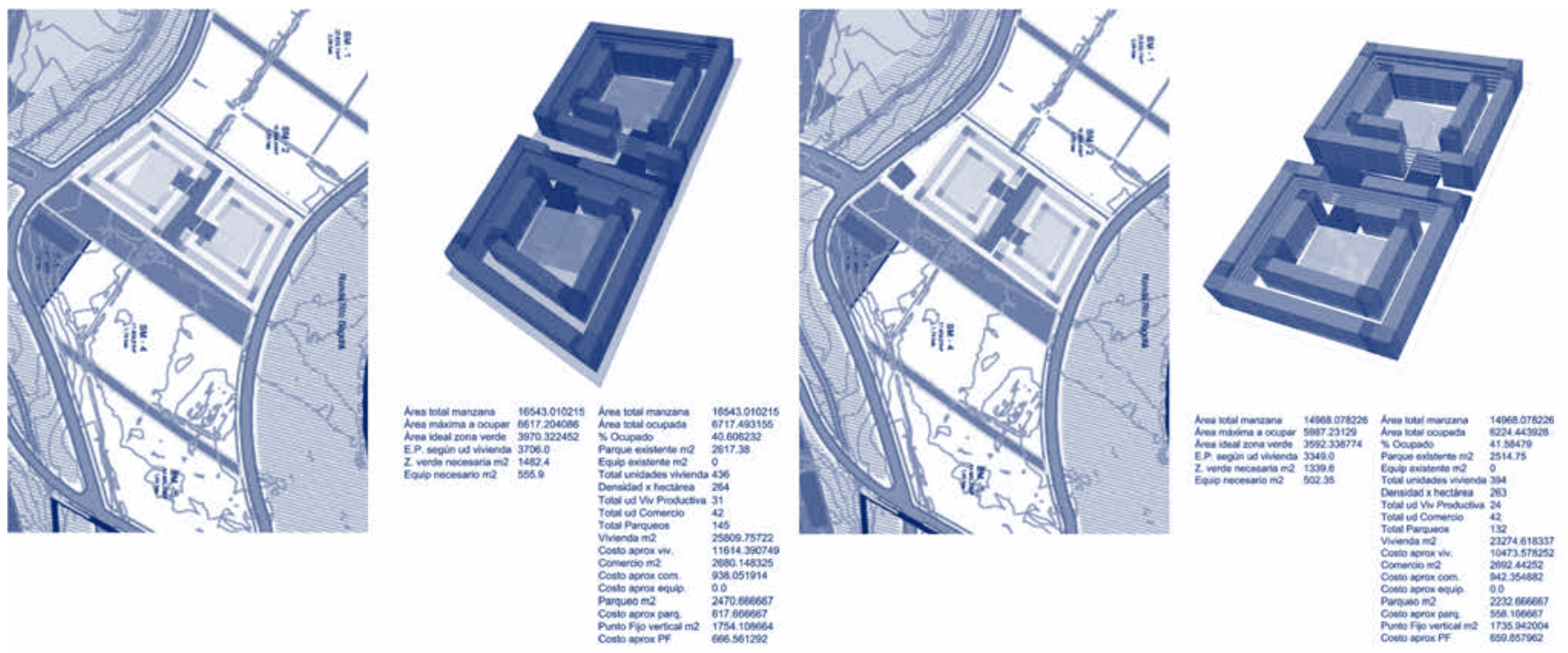

Figura 4: Escenario paramétrico final y su posterior desarrollo en detalle. Escala de agrupación y de unidad. Imagen tomada del Documento Técnico de soporte Proyecto 100/100.

6. Definición de un escenario final para su posterior desarrollo a nivel de anteproyecto: Una vez se llega a un consenso en cuanto a un escenario que cumpla con los rangos y con las recomendaciones de los expertos, se procede a la elaboración de toda la documentación de soporte para su entrega final a Prodesa (Figura 4).

Durante todo el proyecto se hicieron reuniones periódicas con temáticas específicas, a las cuales eran invitados los asesores respectivos. De cada reunión se obtenían conclusiones que se llevaban al modelo paramétrico para complementarlo.

\section{El Modelo Paramétrico}

El modelo paramétrico integra alrededor de 100 variables diferentes, las cuales se cruzan constantemente para analizar resultados y llegar a un escenario deseable.

Una gran parte de los parámetros se pueden visualizar de forma gráfica. Sin embargo la visualización se acompaña de datos numéricos (ideales y reales) cuando es necesario, para tener una comprensión y un análisis mucho más integrado (Figura 3).

EL modelado incluyó parámetros de las tres escalas: ciudad, agrupación y unidad. Sus respectivos parámetros interactúan permanentemente y se pueden hacer recorridos de la escala más grande -ciudad- a la escala más pequeńa -unidad-, viendo las diferentes dependencias y afectaciones que se pueden generar al modificar uno u otro parámetro.

Los escenarios iniciales iban evolucionando en complejidad y cantidad de parámetros integrados. En todos los escenarios se buscaba cumplir con el rango que garantizaba calidad, previamente establecido. Cada escenario se sometía a discusión en las reuniones con el grupo asesor y de las conclusiones se modificaba y complejizaba (Figura 2).

En el escenario final se utilizó una tipología tipo claustro ya que por consenso, era la más apropiada para favorecer y propiciar la calle como punto de encuentro y de desarrollo de la vida cotidiana, y para favorecer la actividad comunal en espacios interiores (Figura 4). Esto evidencia metodológicamente la presencia e integración de variables convertidas a parámetros y variables de diseño más 'convencionales' en un mismo proceso.

\section{Resultados}

La hipótesis planteada inicialmente, fue verificada en la medida que el modelo paramétrico definitivo, efectivamente permitió la integración de una considerable cantidad de variables de diferente índole, con las cuales se hicieron análisis compuestos a partir de datos y modificaciones en tiempo real, para finalmente llegar a un escenario de pre-factibilidad de proyecto, en donde todos los asesores aportaron para su definición.

El modelo paramétrico fue útil en el desarrollo y discusión del proyecto ya que permitía analizar diferentes escenarios preliminares, en los cuales se iban descartando aspectos e integrando otros, facilitando el proceso de toma de decisiones.

La visualización de datos en forma gráfica ayudó a generar una discusión más estructurada entre los asesores del proyecto. El modelo permite recrear diferentes escenarios arrojando información relevante para la toma de decisiones. La información obtenida del modelo puede considerarse como BIM (Eastman, 2011).

El modelo permite hacer modificaciones y adaptar el código a especificidades tanto de observaciones hechas por los asesores como a características propias de la forma de trabajo de Prodesa. En este sentido el código es flexible y puede complementarse o adaptarse a las condiciones de cada proyecto (Figura 3).

\section{Debate}

Proyecto 100/100, más que un proyecto específico de vivienda, es una metodología de diseño que busca mostrar cómo se pueden integrar el tema de calidad, desde el inicio del proceso de diseńo (Figura 4). Uno de los productos finales del convenio Arquitectura Uniandes-Prodesa es el modelo paramétrico, el cual puede ser aplicado a otros escenarios de posible vivienda nueva, recogiendo 
y reutilizando toda la experiencia adquirida en este proyecto específico, donde desde las primeras discusiones y decisiones, se estaría integrando la condición de calidad, a partir de los parámetros trabajados (Figura 3). Este sería un valor agregado para Prodesa como empresa constructora, que le podría ayudar a estructurar mejor sus proyectos en etapas iniciales y que finalmente, beneficiaría al usuario garantizando que los proyectos de vivienda, desde el inicio, incluyan la discusión de calidad en las diferentes escalas.

El modelado paramétrico permite generar aplicaciones adaptadas a requisitos específicos relacionados, ya sean con un proyecto o con una metodología de trabajo. Esto permite adaptar el código a cada condición y escenario específico, y a la información que se espera trabajar y obtener, convirtiendo cada código en un modelo de información de proyecto (BIM).

Ya que la complejidad de cada modelo paramétrico dependerá de las expectativas a alcanzar, el presupuesto para su desarrollo se puede ir administrando y desembolsando según metas específicas. Esto puede llegar a motivar a empresas de diferente tamańo, a implementar modelado paramétrico en sus procesos, ya que podrían invertir poco en principio e ir complejizando los códigos para mejorar procesos y resultados, con inversiones graduales. Podrían decidir también aplicar el modelado paramétrico a etapas específicas de diseño de proyecto (ej. Aplicarlo sólo en la etapa de pre-factibilidad) y trabajar el resto de etapas de forma más convencional. Es aquí donde puede inferirse que esta metodología, puede adaptarse a condiciones locales y específicas de la industria de la construcción.

El manejo de herramientas específicas para modelado paramétrico, y su aplicación al interior de una empresa, requiere la capacitación de un equipo de trabajo, que sea capaz de identificar las necesidades y oportunidades al momento de desarrollar un proyecto arquitectónico. Esto requiere una inversión inicial que garantizaría que el trabajo realizado tenga replicabilidad. De lo contrario, este tipo de experiencias quedan como experiencias aisladas sin mayor impacto en los procesos internos de una empresa constructora.

EL principal problema de la implementación de este tipo de tecnología, es la zona de confort pre-establecida en procesos que llevan un tiempo considerable ejecutándose (así tengas problemas de eficiencia y resultados) al interior de las empresas, que genera una resistencia al cambio.

En ese sentido, Proyecto 100/100 visto como una experiencia de trabajo entre academia y sector productivo, ayuda a evidenciar las ventajas de implementar nuevas metodologías en el proceso de diseño de proyectos de arquitectura, ratificando el beneficio del trabajo entre ambos actores.

\section{Referencias}

Eastman, C. (2011). BIM handbook: a guide to building information modeling for owners, managers, designers, engineers and contractors. New Jersey. John Wiley \& Sons, Inc.

Documento Técnico de Soporte Proyecto 100/100. (2013). Documento interno de trabajo.

Kolarevic, Branko. (2005). Architecture in the digital age: design and manufacturing. New York- London. Taylor \& Francis.

Montaner, J. (2010). De-arq, Revista de Arquitectura de la Universidad de los Andes, No. 6, p. 82-99.

ONU. Oficina del alto comisionado para los derechos Humanos (2010). El derecho humano a una vivienda adecuada (Folleto informativo no. 21).

Steele, J. (2001).Arquitectura y revolución digital. Barcelona: Gustavo Gili.

Velandia, D. (2009). TIC's y los procesos de enseñanza-aprendizaje en arquitectura. De-arq, Revista de Arquitectura de la Universidad de los Andes, No. 5, 166-175.

Villanueva, P. (2004). Transición en la era digital, en la modelización y representación arquitectónica. Diseño y Sociedad, 1 (17), 70-73. 